\title{
Increased risk of suicidal ideation among French women: the mediating effect of lifetime sexual victimisation. Results from the nationally- representative 2017 Health Barometer survey.
}

Fabienne El-Khoury ${ }^{1}$, Meryl Puget ${ }^{2}$, Christophe Leon ${ }^{3}$, Enguerrand du Roscoat ${ }^{3}$, Annie Velter 3, Nathalie Lydié ${ }^{3}$, Audrey Sitbon ${ }^{3}$

- ${ }^{1}$ Sorbonne Université, INSERM UMRS_1136, Institut Pierre Louis d'Épidémiologie et de Santé Publique, Paris, France. fabienne.khoury@inserm.fr.

- $\quad{ }^{2}$ Clinical Psychologist, Paris, France.

- $\quad{ }^{3}$ French National Public Health Agency, Santé Publique France, Saint-Maurice, France. 


\section{Abstract}

\section{Purpose}

Sexual victimisation has been associated with suicidal ideation, especially among women; however data on this association from a large sample of general population is surprisingly limited. Also, no study quantifies sex-differences in the effect of sexual victimisation on suicide risk.

\section{Methods}

We used data from the French Health Barometer, a general population phone survey, which recruited 25,319 adults aged 18 to 75 years in 2017.

Data were weighted to be representative of the French adult population. Three outcomes were examined a) suicidal ideation in the preceding year, b) suicidal imagery (having thought about how to commit suicide), and c) suicide attempt in the preceding year. We conducted adjusted mediation analyses, using the counterfactual approach, to evaluate the contribution that lifetime sexual victimisation has in the association between sex and suicide risk.

\section{Results}

Women were around five times more likely to have experienced lifetime sexual violence $(9.1 \%$ vs $1.9 \%)$, and were more at risk of any suicidal ideation (Ora $=1.20(95 \% \mathrm{CI}$ : 1.07-1.36)) and suicidal imagery (Ora=1.39 (95\%CI: 1.20 -1.61)), but not suicide attempt compared to men in adjusted analysis. In mediation analysis; sexual victimisation explained $49 \%$ and $40 \%$ of the increased risk women have compared to men in suicidal ideation and suicidal imagery respectively. 


\section{Conclusions}

Sexual violence is more prevalent among women and explains a substantial share of sexdifference in suicide risk. Our findings reiterate the importance of the prevention of sexual violence and an adequate care for victims, especially women, in public health and mental health policies and initiatives.

\section{Keywords:}

Suicide risk, sex-differences, sexual violence, mediation analysis. 


\section{Introduction}

2 Sexual violence against women is endemic. In a EU-wide survey published in $2014,11 \%$ of

3 women had declared experiencing some form of sexual violence since the age of 15 (European

4 Union Agency for Fundamental rights 2014). These forms of violence comprised of forced sexual

5 intercourse, attempted forced intercourse and other unwanted or coerced sexual activity. Further,

$612 \%$ of surveyed women reported having experienced some form of sexual violence by an adult

7 before the age of 15 .

8 The experience of sexual violence is a traumatic event which can lead to stress, fear, shame, and

9 isolation, which, in turn, may lead to mental health problems (Jina and Thomas 2013). In fact, the

10 ecological model of the effect of the impact of sexual assault on women's mental health stipulates

11 that sexual violence could lead to self-blame, due to individuals internalising victim-blaming

12 societal myths, which leads to negative self-appraisals (Campbell et al. 2009). Victims of sexual

13 violence are also more likely to experience multiple short and long term consequences, such as

14 post-traumatic stress disorder (PTSD), anxiety, and depression(Au et al. 2013).

15 In fact, sexual violence has been shown to predict the development of PTSD among women in

16 general population more strongly than any other trauma, including physical violence and serious

17 illness (Creamer et al. 2001; Pietrzak et al. 2011). Further, women are generally twice more likely

18 than men to develop PTSD after traumatic events, such as rape, and their symptoms also tend to

19 last longer (Health (UK) 2005). PTSD symptoms include flashbacks where victims relive the

20 trauma over and over, nightmares, and repetitive and distressing images or sensations (nhs.uk

21 2018).This disorder could be chronic, persisting for an extended period of time, and associated

22 with elevated risk of suicidal ideation and suicide attempts (Krysinska and Lester 2010; Pietrzak

23 et al. 2011). 
24 Further, in one study, lifetime suicide risk was shown to be up to three times higher among

25 young adults who have experienced sexual violence compared to those who have not been

26 sexually victimised (Mondin et al. 2016). However, data on the association between sexual

27 violence and adults' suicide risk in general population is surprisingly limited.

28 Moreover, to our knowledge, no study has quantified the mediating effect of past sexual assault

29 on suicide behaviour in a large nationally-representative sample of adults. Nonetheless,

30 quantifying the effect of sexual victimisation on suicidal ideation could be of major importance in

31 advocacy for public policies, and in improving treatment and outcomes for survivors of sexual

32 violence. Therefore, in this analysis, we test whether lifetime sexual violence is more prevalent

33 and is linked with a higher suicidal ideation risk among women compared to men, and quantify

34 its mediating role in the association between sex and suicidal ideation and behaviour.

\section{Methods}

\section{Study design and recruitment}

37 We used data from the "Health Barometer" (Baromètre Santé) 2017 a cross-sectional phone survey, which recruited a nationally-representative sample of French adults aged 18 to 75 years in

39 2017(Equipe Baromètre santé 2017).

40 The survey was commissioned by the French national public health agency (Santé Publique

41 France) and carried out by a polling institute (Ipsos), which used a two-stage random sampling

42 methodology (telephone household, respondent) to recruit participants. Randomly-generated

43 mobile and landline phone number lists were used to call participants up to 40 times using a computer-assisted telephone interviewing (CATI) system. In households reached by landline, one 
45 participant was randomly selected by the CATI system according to the Kish method (Kish 1949).

46 Phone interviews lasted 30 minutes on average, and participation rate was $48.5 \%$.

\section{Ethics}

48 The study protocol was registered in the French Commission on Information Technologies and

49 Liberties (Commission Nationale Informatique et Libertés) platform.

\section{Measures}

51 Suicidal ideation, suicidal imagery, and suicide attempts in the last year

52 Suicidal ideation during the preceding 12 months was examined with the question: 'In the past 12

53 months, have you considered suicide?' (any suicidal ideation yes/no). Participants who reported

54 having suicidal ideation in the last year were also asked if they ever imagined how they would

55 commit suicide (suicidal imagery yes/no) and whether they attempted suicide in the preceding

56 year (suicide attempt yes/no).

57 Experience of lifetime physical sexual violence

58 Respondents were asked whether they had ever been victim of sexual violence ("During your

59 lifetime, have you ever been forced to perform or receive sexual acts ("touching"), or have you

60 ever been forced to have sex against your will?" Yes/No/ does not wish to reply). Participants

61 who reported being victims of physical sexual violence were asked about the time of the first

62 sexual assault. 
63 We then created the variable "experience of lifetime sexual violence" that distinguished

64 participants who had been sexually victimised at least once before the year preceding the study

65 (Yes), from all other participants (No).

\section{Socio-demographic characteristics and other covariates}

67 We adjusted for known risk factors for suicidal ideation and mental health problems in

68 multivariate analysis. We tried to limit collider bias by not adjusting for variables that are likely

69 causally influenced by lifetime sexual violence or mental health problems,(Richiardi et al. 2013)

70 and variables on the pathway(s) between sex and suicide risk.

71 Covariates therefore included sex, age, household monthly income $(<1500 €$ yes/no), whether they

72 had any chronic illness (“do you have a chronic or long-term illness or health problem?" yes/no),

73 and whether they ever lost a parent or a loved one (yes/no). Based on other questions, we were

74 also able to create and include in our models dichotomous covariates for nationality (French by

75 birth yes/no), living in a couple (yes/no), and whether they were victim of verbal and/or physical

76 (not including sexual) violence in the last year (yes/no).

77 Also, participants were classified as belonging to the "sexual minority" group if he or she had

78 ever had a same-sex sexual relationship, or if he or she identified as lesbian, gay, or bisexual.

79 Participants who did not identify as either heterosexual or any of the other mentioned categories

80 were also classified as belonging to the sexual minority group.

\section{Statistical analyses}

82 Descriptive analyses were weighted based on the probability of being solicited through the Kish

83 method (that is the ratio of the number of eligible individuals to the number of telephone lines in

84 a household), and to match the structure of the French population of 2016 with respect to sex, age 
85 groups, region of residency, urban unit size, household size and education level, using data from the National Institute of Statistics and Economic Studies (INSEE) (INSEE 2016).

89 We examined the contribution of lifetime sexual violence to the sex and suicidal behaviour

90 relationship based on the causal diagram presented in supplementary Figure 1. We hypothesised

91 that sex (being a woman) is associated directly and indirectly to suicidal behaviour, and that

92 lifetime sexual assault acts as a mediator in this association.

93 To test our hypotheses, we used multivariate logistic regression on data with complete

94 observations; we first examined the following associations:

95 1) sex and suicide ideation and behaviour outcomes (separately for each outcome),

96 2) sex and lifetime physical sexual victimisation,

97 3) suicide ideation and behaviour outcomes and lifetime physical sexual victimisation.

98 Attenuated associations between sex and suicidal behaviour were expected after adjustment for 99 sexual violence, which would indicate a potential mediating role of the latter.

100 Second, we performed a formal mediation analysis by using the counterfactual approach, also 101 adjusting for the listed covariates. Analyses were also carried out on complete observations. This method allows the identification of direct and indirect effects of sex (our exposure) on mental

103 health in a single model. The exposure, mediator and outcome were dichotomized and all covariates were either binary or continuous. Direct and indirect effects of sex and the proportion

105 of the association with mental health outcomes mediated by physical sexual victimisation were estimated using the method described by VanderWeele and Vansteelandt.(VanderWeele and

107 Vansteelandt 2010) Logistic regressions were used since outcomes are rare $(<10 \%)$, and exposure- 
mediators were tested for mediation analysis. The proportion mediated through mediator was calculated on the risk difference scale. The proportion mediated was calculated using the estimated natural indirect effect (NIE) and total effect (TE) : $(\ln (\mathrm{ORNIE}) / \ln (\mathrm{ORTE})] \times 100 \%$

111 (Menvielle et al. 2016).

112 Multivariate and mediation analysis were then repeated using 'childhood sexual victimisation' as 113 the exposure.

114 All analyses were conducted with SAS 9.4. Mediation analyses were implemented using the SAS macro “\%mediation" developed by Valeri and VanderWeele (Valeri and VanderWeele 2013).

\section{Results}

117 Around $6 \%$ of the population reported lifetime sexual victimisation, with missing data on 89

118 participants for this variable: 72 (weighted percentage: $0.33 \%$ ) refused to answer this question and 17 participants $(0.10 \%)$ replied with "I don't know". Women were around five times more likely to be victim of physical sexual violence compared to men ( $9.1 \%$ vs $1.9 \%)$. The median age of the first occurrence of sexual victimisation was $12(\mathrm{IQR}=9$; mean $=13.4(\mathrm{sd}=8))$. The main characteristics of our weighted sample (unweighted $n=25319$ ) are presented in Table 1, by sex. In bivariate analysis, suicidal ideation, suicidal imagery, and suicide attempt in the last year were more prevalent among women compared to men. suicidal ideation $(\mathrm{ORa}=1.20(95 \% \mathrm{CI}, 1.07-1.36)$, and suicidal imagery $(\mathrm{ORa}=1.39(95 \% \mathrm{CI}, 1.20-$ 1.61) in the last year. However women were not more likely than men to have attempted to commit suicide in the preceding year after adjusting for potential confounders $(\mathrm{ORa}=1.38$ (95\% CI, $0.89-2.06)$, though this outcome was especially rare with less than $0.5 \%$ of participants reporting it. Lifetime sexual violence was strongly associated with all outcomes (table 3); 
131 therefore mediation analyses for the two outcomes: suicidal ideation and suicidal imagery in the

132 last year were possible.

133 Mediation analysis

134 No exposure-mediator interaction was statistically significant; therefore it was not included in

135 our models. The results of the multivariate mediation analysis are presented in Figure $\mathbf{1}$ and

136 supplementary table $1:$.

137 After taking into account lifetime sexual violence, the natural direct effect between sex and

138 suicidal ideation was not significant, with an ORa=1.12 (95\% CI, 0.98- 1.28).Further, the natural

139 indirect effect between sex (being a woman compared to men) on suicidal ideation mediated by

140 lifetime physical sexual violence was significant (ORa=1.12 (95\% CI, 1.09- 1.15)). Overall, we

141 estimated that $49 \%$ of the increased risk of suicidal ideation in the preceding year women have

142 compared to men is mediated by lifetime sexual assault.

143 For the outcome suicidal imagery, the direct effect of sex was still statistically significant after

144 controlling for sexual victimisation $(\mathrm{ORa}=1.20(95 \% \mathrm{CI}, 1.03-1.41))$, as was the indirect effect

$145(\mathrm{ORa}=1.13[95 \% \mathrm{CI} 1.10-1.17])$. The proportion of effect mediated by lifetime physical sexual

146 violence was $40 \%$.

\section{Discussion}

\section{Key results}

149 In a large nationally-representative sample of French general population, we found that women

150 are five times more likely to have experienced lifetime sexual violence, and are more at risk of

151 any suicidal ideation and imagery of suicide in the preceding year compared to men. Our study 
152 adds to prior data by quantifying the substantial contribution (around 50\%) of lifetime sexual

153 victimisation to women's increased likelihood of suicidal ideations compared to men. Our

154 findings reiterate the importance of the prevention of sexual violence and an adequate care for 155 sexual assault victims, especially women, in public health and mental health policies and 156 initiatives.

\section{Interpretation}

158 A history of sexual abuse has been extensively linked with suicidal ideations, suicide attempts as well as completed suicide in the literature among both men and women (Chen et al. 2010; Gradus et al. 2012). Experience of physical sexual violence is a traumatic event that could lead to PTSD and feelings of worthlessness, in turn these two symptoms could last for decades and lead to suicidal ideation (Jeon et al. 2014). Further, sexual violence, especially in a victim blaming culture, could also generate self-blame, shame, and anticipatory stigma that would halt survivors' disclosure and help seeking, and are linked with depression, psychological distress, and maladaptive coping that could lead to suicidal ideation (Kennedy and Prock 2018).

166 Our findings suggest that sex-difference in suicidal ideation and imagery of suicide may be partly explained by women's greater exposure to sexual violence than men. There is evidence that increased risk in depression and anxiety disorders among women compared to men are also strongly linked to sexual victimisation (Chen et al. 2010). It is also possible that the psychological effect of sexual violence among women is stronger due to a more important risk of revictimisation throughout their life (Najdowski and Ullman 2011).

172 We did not find any effect between sex and suicide attempt in the preceding year. This may be 173 partly due to small statistical power due to the low rate of suicide attempt in the last year $(0.5 \%)$.

174 This low rate is however comparable to others found in other countries (Johnston et al. 2009; 
175 Olfson et al. 2017). We chose to examine rates of suicide attempt in the last year, and not lifetime

176 events, in order to respect temporality and make sure that sexual violence occurred before suicide

177 risk.

178 Possible biological mechanisms

179 Experience of sexual violence during childhood has been linked with structural abnormalities in

180 the brain, such as diminished volume in several cerebral regions. (Walsh et al. 2012) It has also

181 been linked with the development of emotion dysregulation, and epigenetic modification. These

182 hypothesized biological mechanisms could explain the risk of negative mental health outcomes

183 among sexual violence victims.

\section{$184 \quad$ Limitations and strengths}

185 Certain methodological aspects of our study warrant comments. One of the limitations is that this survey was cross-sectional and retrospective, which might imply potential memory bias in the recall of early experiences. Also, the recall of experiences of sexual abuse could be underreported, in part because of recall or desirability bias. Not everyone who has suffered victimisation might recall the experience, identify it as such, or be willing to report it, which would result in underreporting (Wolf and Nochajski 2013). Further, data was unavailable for 89 participants for this variable, although this likely did not result in significant bias given the large sample size. Also, desirability bias could also be more important among men where sexual victimisation is more taboo than among women. However, the study was conducted anonymously by telephone, which should limit the extent of this type of bias; also, these experiences may be difficult to forget which might explain their lingering psychological consequences.

196 Moreover, we were unable to account for non-suicidal self-injury, and completed suicides due to 197 the study methodology. Nonetheless the rates of completed suicides are highly low ( 15 per 
198100 000) and would have probably not provided sufficient statistical power for multivariate

199 analysis.

200 The strengths of our study include large sample size and a nationally representative sample,

201 which improves the generalizability of our findings. We were also able to limit any bias due to

202 the temporality of exposure and outcomes, since we only included sexual violence that occurred

203 at least one year before the survey. Further, we had data on a range of covariates such as sexual

204 orientation and other violence that allowed adjustment for a wide range of potential confounders.

\section{Conclusion}

206 Our study provides evidence that sexual violence account for a significant share of women's 207 increased risk of suicidal ideation. We add to a substantial number of scientific evidence that

208 links sexual violence to mental health problems. More comprehensive policies need to be put in 209 place to limit sexual violence, especially violence against women. A history of sexual violence 210 should also be investigated when possible in the events of attempted or completed suicide, in 211 order to better quantify the effect if sexual victimisation on suicide risk. 


\section{Compliance with Ethical Standards}

\section{Funding}

Data came from the 2017 "Baromètre santé", a study conducted and funded by the French Public Health Agency (Santé Publique France).

\section{Conflict of Interest:}

The authors declare that they have no conflict of interest.

\section{Statement of human rights}

This study uses data collected in a repeated cross-sectional survey for official statistics (inpes.santepubliquefrance.fr/Barometres/index.asp).

All procedures performed in the study involving human participants were in accordance with the ethical standards of the national ethics committee "Commission Nationale de l'Informatique et des Libertés" (CNIL; National commission for liberty and informatics), and with the 1964 Helsinki declaration and its later amendments or comparable ethical standards. The original data collection protocol for the repeated surveys and the questionnaire were approved by the CNIL : $\mathrm{N}^{\circ} 1,179,915$.

\section{Informed consent}

Informed consent was obtained from all individual participants included in the study (orally). 


\section{References}

Au TM, Dickstein BD, Comer JS, et al (2013) Co-occurring posttraumatic stress and depression symptoms after sexual assault: A latent profile analysis. J Affect Disord 149:209-216. https://doi.org/10.1016/j.jad.2013.01.026

Campbell R, Dworkin E, Cabral G (2009) An Ecological Model of the Impact of Sexual Assault On Women's Mental Health. Trauma Violence Abuse 10:225-246. https://doi.org/10.1177/1524838009334456

Chen LP, Murad MH, Paras ML, et al (2010) Sexual Abuse and Lifetime Diagnosis of Psychiatric Disorders: Systematic Review and Meta-analysis. Mayo Clin Proc 85:618-629. https://doi.org/10.4065/mcp.2009.0583

Creamer M, Burgess P, Mcfarlane AC (2001) Post-traumatic stress disorder: findings from the Australian National Survey of Mental Health and Well-being. Psychol Med 31:. https://doi.org/10.1017/S0033291701004287

Equipe Baromètre santé (2017) Baromètre santé 2017. Méthode. Santé Publique Fr 24

European Union Agency for Fundamental rights (2014) Violence against women: An EU-wide survey: Main results. FRA, European Union Agency for Fundamental Rights

Gradus JL, Qin P, Lincoln AK, et al (2012) Sexual Victimization and Completed Suicide Among Danish Female Adults. Violence Women 18:552-561. https://doi.org/10.1177/1077801212453141

Health (UK) NCC for M (2005) Post-traumatic stress disorder. Gaskell

INSEE (2016) La population totale en 2016 - Activité, emploi et chômage en 2016. https://www.insee.fr/fr/statistiques/2841321?sommaire=2841366. Accessed 7 Dec 2018

Jeon HJ, Park J-I, Fava M, et al (2014) Feelings of worthlessness, traumatic experience, and their comorbidity in relation to lifetime suicide attempt in community adults with major depressive disorder. J Affect Disord 166:206-212. https://doi.org/10.1016/j.jad.2014.05.010

Jina R, Thomas LS (2013) Health consequences of sexual violence against women. Best Pract Res Clin Obstet Gynaecol 27:15-26. https://doi.org/10.1016/j.bpobgyn.2012.08.012

Johnston AK, Pirkis JE, Burgess PM (2009) Suicidal Thoughts and Behaviours Among Australian Adults: Findings from the 2007 National Survey of Mental Health and Wellbeing. Aust N Z J Psychiatry 43:635-643. https://doi.org/10.1080/00048670902970874

Kennedy AC, Prock KA (2018) "I Still Feel Like I Am Not Normal": A Review of the Role of Stigma and Stigmatization Among Female Survivors of Child Sexual Abuse, Sexual Assault, and Intimate Partner Violence. Trauma Violence Abuse 19:512-527. https://doi.org/10.1177/1524838016673601

Kish L (1949) A Procedure for Objective Respondent Selection within the Household. J Am Stat Assoc 44:380-387. https://doi.org/10.1080/01621459.1949.10483314 
Krysinska K, Lester D (2010) Post-Traumatic Stress Disorder and Suicide Risk: A Systematic Review. Arch Suicide Res 14:1-23. https://doi.org/10.1080/13811110903478997

Menvielle G, Franck J, Radoï L, et al (2016) Quantifying the mediating effects of smoking and occupational exposures in the relation between education and lung cancer: the ICARE study. Eur J Epidemiol 31:1213-1221. https://doi.org/10.1007/s10654-016-0182-2

Mondin TC, Cardoso T de A, Jansen K, et al (2016) Sexual violence, mood disorders and suicide risk: a population-based study. Ciênc Saúde Coletiva 21:853-860. https://doi.org/10.1590/1413-81232015213.10362015

Najdowski CJ, Ullman SE (2011) The effects of revictimization on coping and depression in female sexual assault victims. J Trauma Stress 24:218-221. https://doi.org/10.1002/jts.20610

nhs.uk (2018) Post-traumatic stress disorder (PTSD). In: nhs.uk. https://www.nhs.uk/conditions/post-traumatic-stress-disorder-ptsd/. Accessed 29 May 2019

Olfson M, Blanco C, Wall M, et al (2017) National Trends in Suicide Attempts Among Adults in the United States. JAMA Psychiatry 74:1095. https://doi.org/10.1001/jamapsychiatry.2017.2582

Pietrzak RH, Goldstein RB, Southwick SM, Grant BF (2011) Prevalence and Axis I comorbidity of full and partial posttraumatic stress disorder in the United States: Results from Wave 2 of the National Epidemiologic Survey on Alcohol and Related Conditions. J Anxiety Disord 25:456-465. https://doi.org/10.1016/j.janxdis.2010.11.010

Richiardi L, Bellocco R, Zugna D (2013) Mediation analysis in epidemiology: methods, interpretation and bias. Int J Epidemiol 42:1511-1519. https://doi.org/10.1093/ije/dyt127

Valeri L, VanderWeele TJ (2013) Mediation analysis allowing for exposure-mediator interactions and causal interpretation: Theoretical assumptions and implementation with SAS and SPSS macros. Psychol Methods 18:137

VanderWeele TJ, Vansteelandt S (2010) Odds ratios for mediation analysis for a dichotomous outcome. Am J Epidemiol 172:1339-1348

Walsh K, Galea S, Koenen KC (2012) Mechanisms Underlying Sexual Violence Exposure and Psychosocial Sequelae: A Theoretical and Empirical Review. Clin Psychol Sci Pract 19:260275. https://doi.org/10.1111/cpsp.12004

Wolf MR, Nochajski TH (2013) Child Sexual Abuse Survivors with Dissociative Amnesia: What's the Difference? J Child Sex Abuse 22:462-480. https://doi.org/10.1080/10538712.2013.781094 


\section{Tables}

Table 1: Characteristics of participants in the Health Barometer survey (weighted, \%). France, 2017. Unweighted $n=25,319$

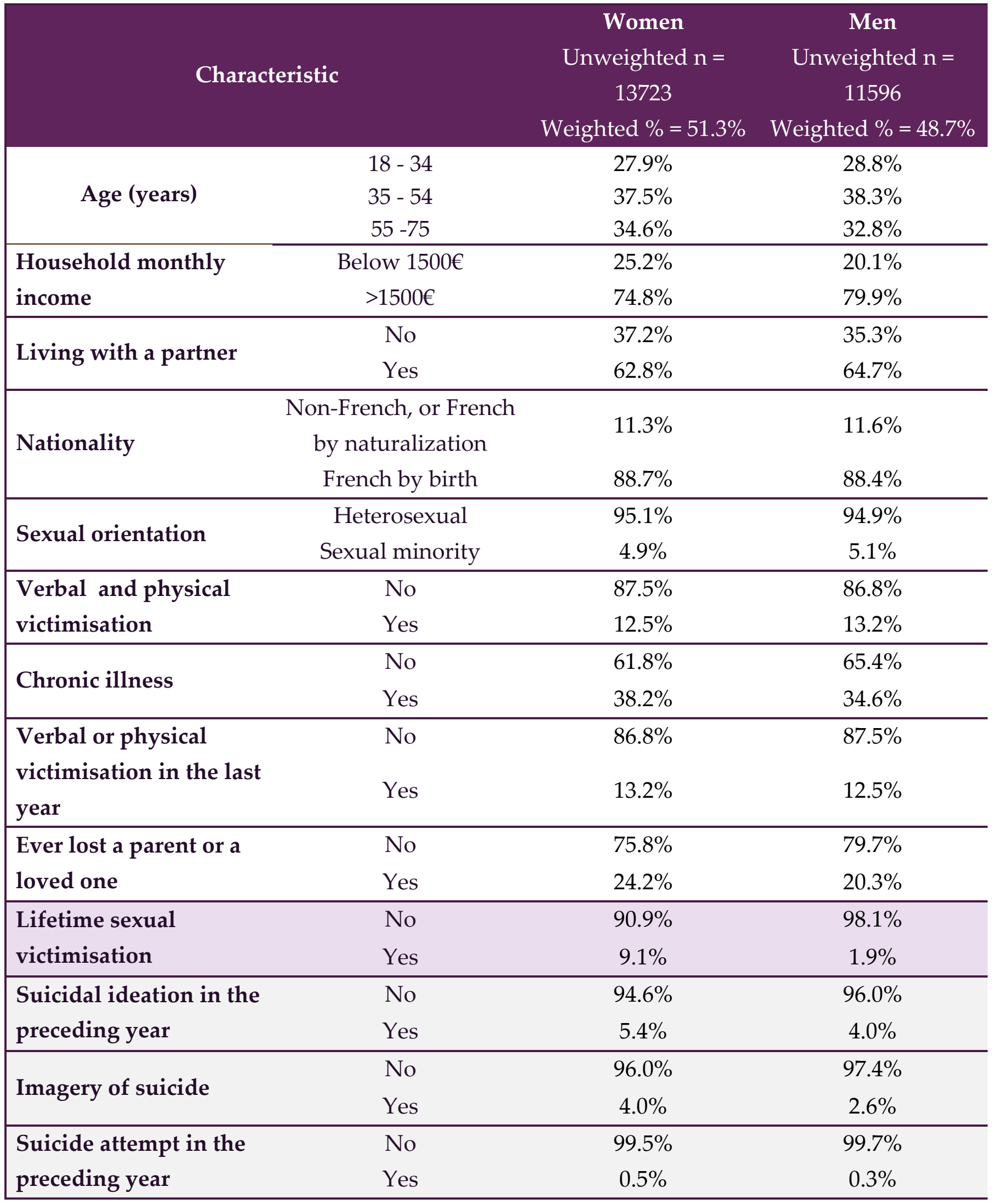


Table 2: Results of three different multivariate logistic models, Adjusted ORs (95\%CI).unweighted n= 24 675, The French

Health Barometer survey, 2017.

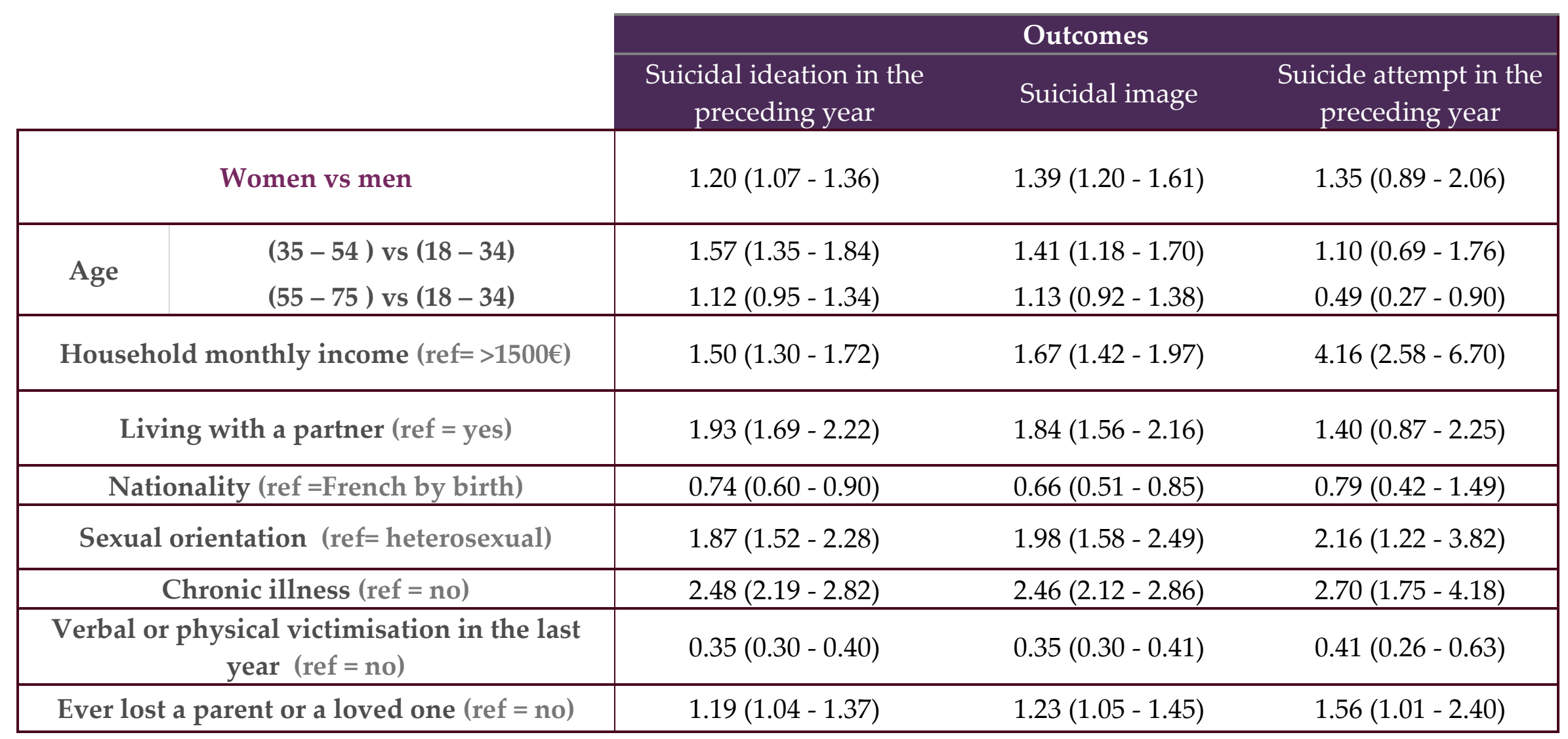


Table 3: Results of three different multivariate logistic models, Adjusted ORs (95\%CI).unweighted n= 24 603, The French

Health Barometer survey, 2017.

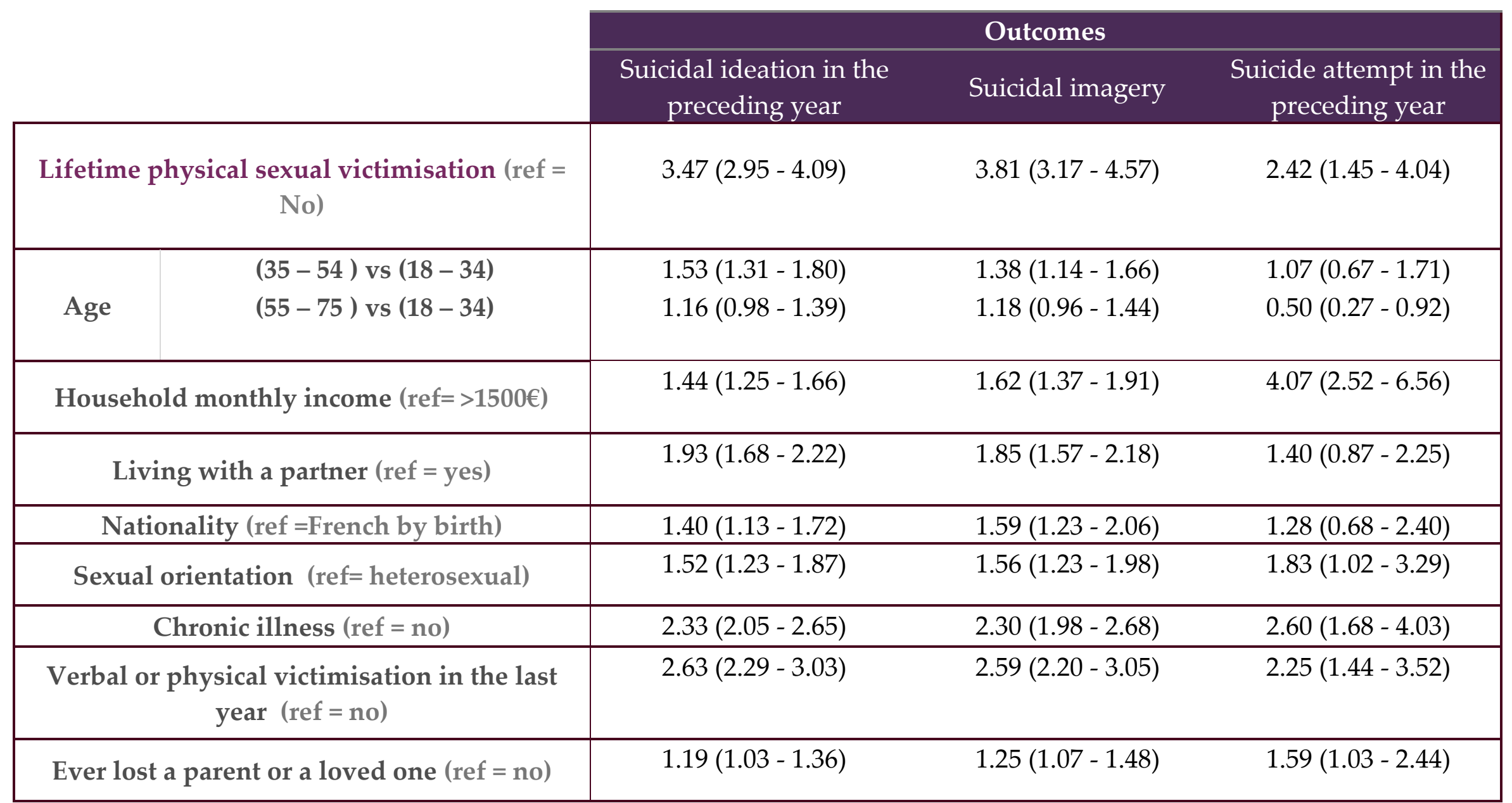




\section{Figure 1}

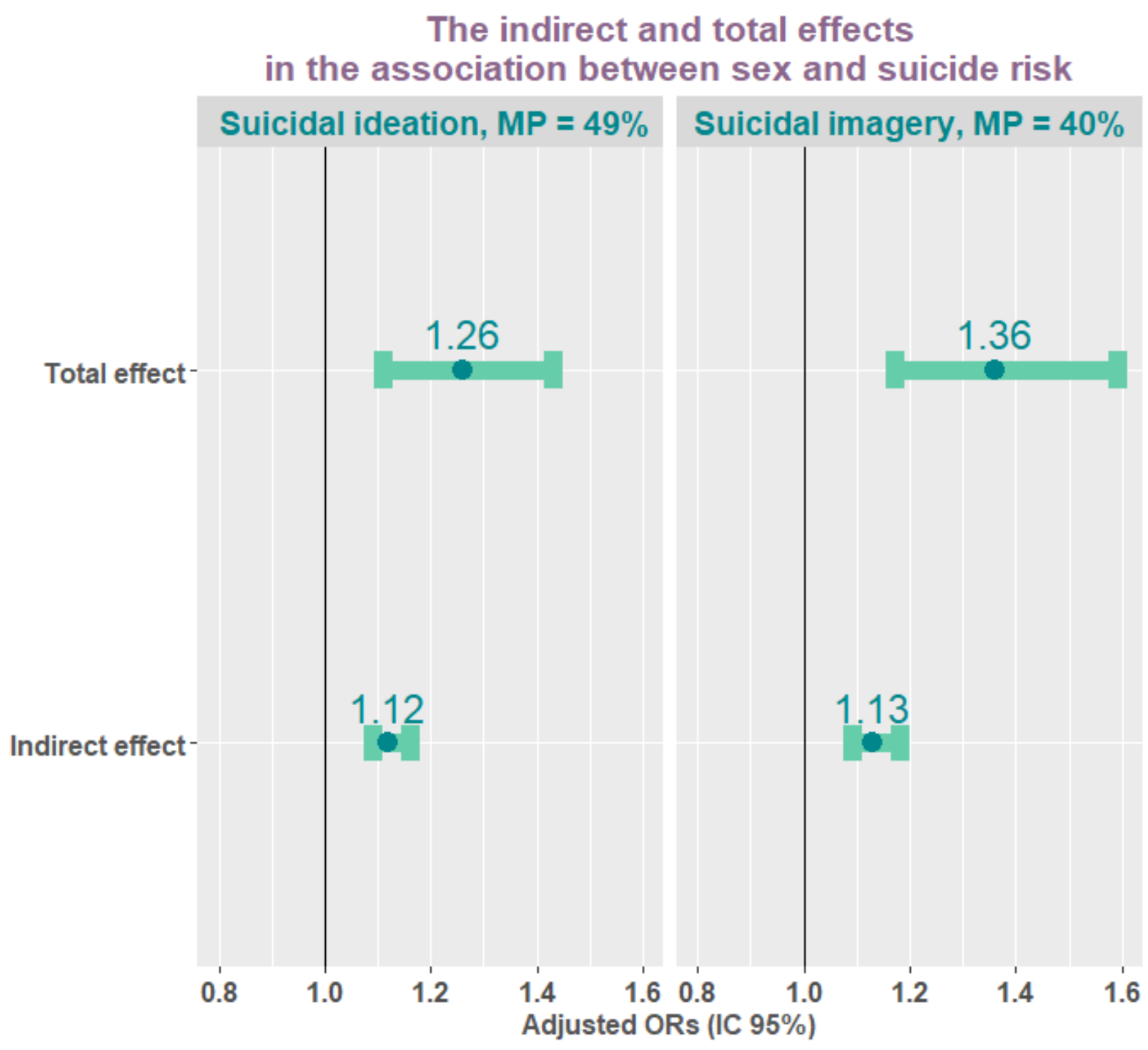

The models are adjusted for sex, age, household income, living with a partner, sexual orientation, physical and verbal violence in the last year, nationality, chronic illness, and having lost a parent or a loved one. $n=24,603$, The French Health Barometer survey, 2017. ${ }^{*} \mathrm{MP}=$ Mediated proportion $=\left[\ln \left(\mathrm{OR}^{\mathrm{IE}}\right) / \ln \left(\mathrm{OR}^{\mathrm{TE}}\right)\right] \times 100 .($ with ie$=$ indirect effect, and te=total effect $)$ 


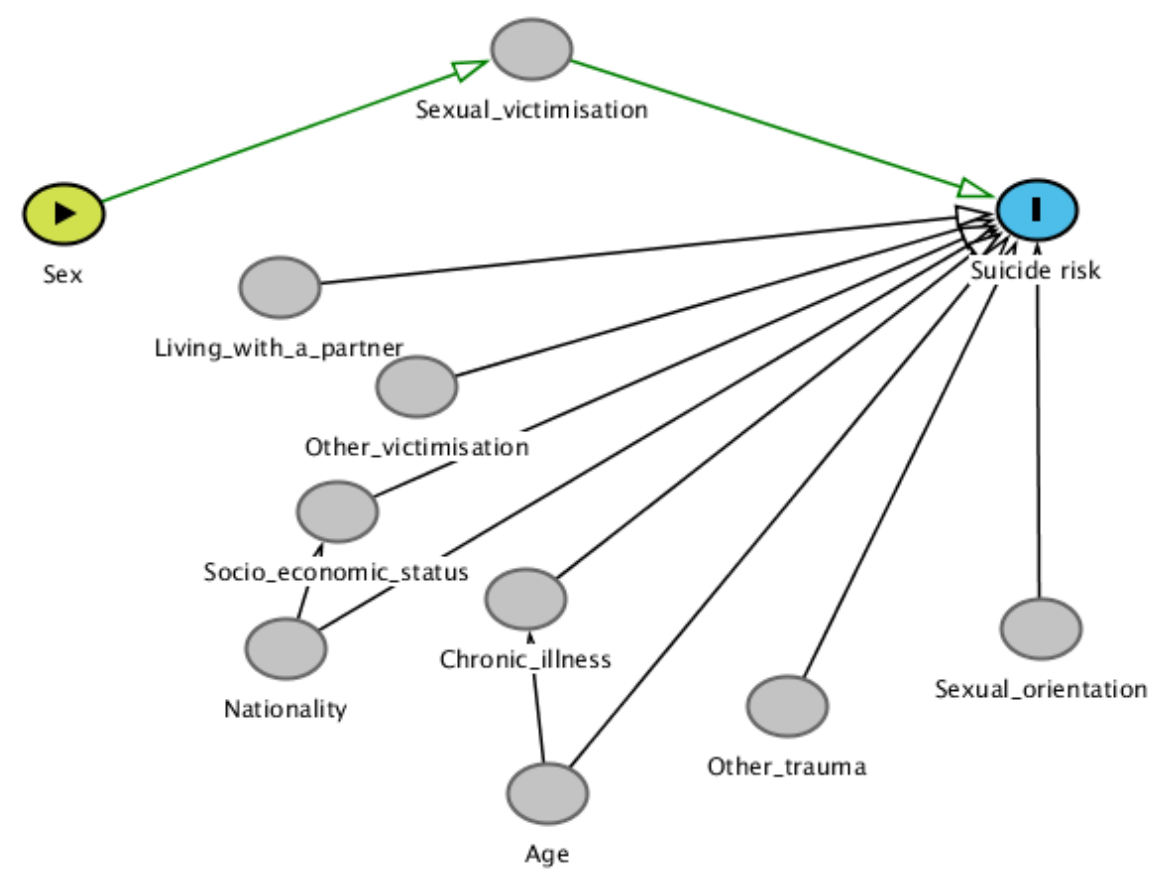

Supplementary Figure 1: Directed acyclic graph for the association between sex and suicide risk, showing the confounders (grey lines) and mediator (green line) used in the final model. Graph done using the web application on daggity.net. http://dagitty.net/mkbfWUS

Supplementary table 1: results of the adjusted mediation analysis.

\begin{tabular}{|c|c|c|c|c|c|c|c|}
\hline & $\begin{array}{c}\text { Direct } \\
\text { effect }\end{array}$ & $\mathbf{p}$ & $\begin{array}{c}\text { indirect } \\
\mathbf{e f f e c t}\end{array}$ & $\mathbf{p}$ & total effect & $\mathbf{p}$ & $\mathbf{M P}$ \\
$\begin{array}{c}\text { Suicidal } \\
\text { ideation in } \\
\text { the }\end{array}$ & $\begin{array}{c}1.12(0.98- \\
1.28)\end{array}$ & 0.08885 & $\begin{array}{c}1.12(1.09- \\
1.15)\end{array}$ & $<0.00001$ & $\begin{array}{c}1.26(1.11- \\
1.43)\end{array}$ & 0.00650 & $49 \%$ \\
$\begin{array}{c}\text { preceding } \\
\text { year }\end{array}$ & & & & & & & \\
\hline $\begin{array}{c}\text { Suicidal } \\
\text { imagery }\end{array}$ & $\begin{array}{c}1.20(1.03- \\
1.41)\end{array}$ & 0.02346 & $\begin{array}{c}1.13(1.10- \\
1.17)\end{array}$ & $<0.00001$ & $\begin{array}{c}1.36(1.17- \\
1.59)\end{array}$ & 0.00130 & $40 \%$ \\
\hline
\end{tabular}

${ }^{*} \mathrm{MP}=$ Mediated proportion $=\left[\ln \left(\mathrm{OR}^{\mathrm{IE}}\right) / \ln \left(\mathrm{OR}^{\mathrm{TE}}\right)\right] \times 100$. (with ie= indirect effect, and te=total effect) 